\title{
Les négociations franco-espagnoles pendant la Guerre de Succession d'Espagne
}

\author{
Lucien BÉLY \\ Université Paris-Sorbonne, France \\ Centre Roland Mousnier UMR 8596 \\ Lucien.Bely@paris-sorbonne.fr
}

Fecha de recepción: 15/05/2013

Fecha de aceptación: 28/06/2013

\begin{abstract}
RESUMEN
Los historiadores han señalado, con razón, los estrechos lazos que han unido las dos monarquías española y francesa durante la Guerra de Sucesión española hasta el punto de que Luis XIV ha podido aparecer como el verdadero Rey de España, en una visión imperial según la cual la rama más antigua guía a la rama más joven. Este estudio tiene como objetivo mostrar la negociación continuada entre los dos cortes: la discusión se hace sobre la base de una relación diaria y a varios niveles. Todo el aparato del Estado ha resistido las injerencias demasiado patentes de los franceses y en particular ha querido limitar la penetración en el mercado, especialmente en la América española. La diplomacia francesa ha tomado también en cuenta las negociaciones con los holandeses y después con los ingleses. Cuando el Congreso se abre en 1712, el rey de Francia todavía habla en nombre de su nieto, al que los aliados no reconocen. Sin embargo, la discusión internacional se torna en un difícil diálogo entre Madrid y Versalles sobre las concesiones de Felipe V y la cesión de Sicilia. Estas complejas relaciones acompañan la evolución histórica de la monarquía española, que mantiene su imperio en el mundo aún abandonando su dominio europeo fuera de la península.
\end{abstract}

Palabras clave: Negociación, renuncia, imperio, monopolio comercial, Tratado, Comercio, Información Económica, Monarquía, Congreso, Embajador, sucesión.

\section{Negotiations between France and Spain during the War of the Spanish Succession}

\begin{abstract}
Historians have rightly pointed out the close ties that have united both Spanish and French monarchies during the War of the Spanish Succession, to the point that Louis XIV of France was able to emerge as the true King of Spain, in an imperial vision whereby the oldest dynastic branch guides the younger. This study aims to show the ongoing negotiations between the two courts: the discussion is made on the basis of a daily relationship at various levels. The entire apparatus of the state has resisted to the blatant interference of the French, particularly in order to limit the market penetration, especially in the Spanish New World. French diplomacy has also taken account of the negotiations with the Dutch and later the British. When the Congress opened in 1712, the King of France still spoke on behalf of his grandson, who was not recognized by the allies. However, the international discussion became a difficult issue between Madrid and Versailles on Philip V's concessions and on the cession of Sicily. These complex relationships accompany the historical evolution of the Spanish monarchy, which maintained its empire in the world even abandoning their European dominions outside the Iberian peninsula.
\end{abstract}

Key words: Negotiation - resignation - Empire - commercial monopoly - Treaty - Trade - Economic Information - Monarchy - Congress - Ambassador - Succession. 
La guerre de Succession d'Espagne voit le roi de France Louis XIV et son petit-fils affronter une large coalition européenne. Aux yeux des alliés, la construction impériale dessinée par les Bourbons, cette somme de puissances, cette transformation de Louis XIV en roi d'Espagne, pour reprendre la formule de José Manuel de Bernardo Ares $^{1}$, aboutit à une impasse et à un échec.

À mesure que l'engagement militaire des puissances se fait plus large, plus difficile, plus coûteux, une réflexion stratégique globale s'impose dans les deux camps et des choix se font jour pour dégager les intérêts essentiels. Les Bourbons défendent la péninsule ibérique ${ }^{2}$, non sans mal, les domaines américains de l'Espagne et les liaisons maritimes entre ces deux ensembles. La Grande Alliance de 1701 permet une collaboration entre les puissances maritimes et l'empereur et prépare une grande offensive combinée, pour pénétrer en Allemagne et en Italie, pour prendre à revers le royaume de France, avec l'espoir d'une révolte des protestants, ainsi qu'en Catalogne, pour conquérir la péninsule Ibérique.

Cette longue guerre suscite des négociations entre les belligérants, chaque camp essayant de briser la coalition qui se dresse face à lui. En 1703, le Portugal et la Savoie signent des traités avec les ennemis des Bourbons. Ce que je souhaite souligner ici, c'est que les discussions existent aussi en permanence entre alliés, en particulier entre la France et l'Espagne ${ }^{3}$.

\section{LAQUESTION COMMERCIALE DANS LES RELATIONS DIPLOMATIQUES}

Alors que la Cour de Madrid semble suivre en aveugle les décisions prises à Versailles, dans la réalité, des tensions fortes marquent cette collaboration diplomatique et militaire. Les réticences de l'administration espagnole vis-à-vis des injonctions françaises s'imposent en particulier dans le domaine commercial.

En effet, la participation des étrangers au commerce hispano-américain malgré le monopole colonial constitue, à la fin du XVIIe siècle, un des enjeux essentiels dans les relations internationales. Les économies européennes alimentent en produits nécessaires le négoce qui reste aux mains des Espagnols, est contrôlé par eux et protégé par la flotte du roi Catholique ${ }^{4}$. L'enquête du consul français Patoulet en 1686 a montré la place de chacun dans le commerce de Cadix (Cadiz) ${ }^{5}$ : si la France vient au

\footnotetext{
1 Bernardo Ares, J. M.: Luis XIV rey de España. De los imperios plurinacionales a los estados unitarios (1665-1714), Madrid, Iustel, 2008.

2 Kamen, H.: The war of succession in Spain, 1700-1715, London, Weidenfeld and Nicolson, 1969; García González, F. (coord.): La Guerra de Sucesión en España y la Batalla de Almansa. Europa en la encrucijada, Madrid, Sílex, 2009; Albareda Salvadó, J.: La Guerra de Sucesión en España (1700-1714), Barcelona, Crítica, 2010.

3 Il convient de se reporter à ce propos à l'ouvrage classique d'Alfred Baudrillart; BAUDRILLART, A.: Philippe Vet la cour de France, Paris, Firmin-Didot, 1890.

4 García-Baquero González, A.: La Carrera de Indias. Histoire du commerce hispano-américain (XVIeXVIIIe siècles); traduction par B. BENNASSAR, Paris, Desjonquères, 1997.

5 Voir Girard, A.: Le Commerce français à Séville et Cadix au temps des Habsbourg. Contribution à l'étude du commerce étranger en Espagne aux XVIe et XVIIe siècles, Paris, De Boccard, 1932; ainsi que les travaux de John Everaert et Michel Morineau.
} 
premier rang, Gênes, les Provinces-Unies, l'Angleterre, Hambourg, et les Pays-Bas espagnols ont un poids certain, l'Espagne ne fournissant que 6\% des marchandises. Les étrangers eux-mêmes sont présents dans les ports espagnols, ainsi les Flamands étudiés par Jan Everaert pour Cadix ${ }^{6}$, ou les Irlandais présents aux Canaries, étudiés par A. Guimerá Ravina?

On sait aussi que les arrivées de métaux précieux ont eu des conséquences capitales pour les économies européennes. La "crise du XVIIe siècle", démontrée par Earl J. Hamilton ${ }^{8}$ et réinterprétée par P. Chaunu ${ }^{9}$, a été l'objet d'une remise en cause par M. Morineau ${ }^{10}$. L'étude de ces phénomènes, en particulier de la place du métal précieux et de la monnaie métallique dans le développement de l'Europe, a donc suscité des controverses fécondes pour la réflexion des historiens, et sans doute pour des économistes. Les contemporains sont convaincus de l'importance cruciale de l'argent américain. Encore, au début du XVIIIe siècle, Mesnager, dit que "c'est presque l'unique source des richesses de l'Europe" 11 . Et en 1710, il écrit encore: "L'Amérique espagnole renferme les grandes sources de l'or et de l'argent qui sont dans le monde. La terre en donne tous les ans 55 ou 60 millions de livres, chacun veut en avoir sa part". Les puissances européennes se mesurent -au sens propre comme au sens figuré- à travers leur participation à ces échanges au sein de l'empire espagnol.

Une telle dimension économique s'introduit donc également dans les négociations européennes, en particulier au début du XVIIIe siècle, lors de la guerre de Succession d'Espagne qui déclenche une guerre économique ${ }^{12}$. L'avidité des pays européens face à la Carrera a un lien avec les idées du temps: au XVIIe siècle, les États, en tant que tels, ont l'ambition de se charger eux-mêmes de missions commerciales. Ainsi, le travail de Colbert et de son fils a visé à "nationaliser" le commerce français. Les accords doivent avoir lieu à l'échelon des États, en écartant les initiatives individuelles et dispersées des négociants.

Les puissances maritimes -Angleterre et Provinces-Unies-s'engagent dans le conflit en 1701 pour éviter que la présence d'un Bourbon à Madrid ne favorise une

6 Everaert, J.: De internationale en koloniale Handel der Vlaamse Firma's te Cadiz (1670-1700), Bruges, «de Tempel», 1973.

7 Guimerá Ravina, A., Delgado Barrado, J. M.: "Proyectismo canario y comercio americano: un plan de compañía privilegiada (1753)”, en Lobo Cabrera, M., Martínez Ruiz, E., Suárez Grimón, V. J. (coords.): III Reunión Cientifica de la Asociación Española de Historia Moderna (1994), El Comercio en el Antiguo Régimen, Las palmas de Gran Canaria, 1995, vol.2, pp. 151-162.

8 Hamilton, E. J.: American Treasure and Price Revolution in Spain (1501-1650), Cambridge, Harvard UP, 1934; Hamilton, E. J.: War and Prices in Spain, Cambridge, Harvard UP, 1947.

9 Chaunu, H., Chaunu, P.: Séville et l'Atlantique (1504-1650). Partie statistique, Paris, SEVPEN, 1958; Chaunu, P.: Séville et l'Atlantique (1504-1650). Partie interprétative, Paris, SEVPEN, 1960.

10 Morineau, M.: Incroyables gazettes et fabuleux métaux, Paris, Éditions de la Maison des sciences de l'homme, 1985.

11 Pour tout ce qui concerne Nicolas Mesnager, je me permets de renvoyer à BeLY, L.: Espions et ambassadeurs au temps de Louis XIV, Paris, Fayard, 1990, pp. 576-595, ici p. 582. Il s'agit d'une citation tirée des "Réflexions sur les moyens qui peuvent aider à déterminer les Hollandais à la paix", Archives du Ministère des Affaires étrangères, Correspondance politique Hollande 214, fol. 105-107.

12 BéLy, L.: Les routes de la mer: l'enjeu économique dans les négociations diplomatiques, en BouRILLON, F., Boutry, P., Encreyé, A., Touchelay, B. (dir.): Des économies et des hommes. Mélanges offerts à Albert Broder, Bordeaux, Bière, 2006, pp. 251-258. 
mainmise française sur le commerce espagno ${ }^{13}$. La Grande Alliance prévoit que ces deux puissances conserveront les conquêtes qu'elles pourront faire en Amérique. Le changement dynastique en Espagne peut-il favoriser le négoce français et fermer le marché américain aux autres producteurs européens? L'asiento, le contrat qui permet de fournir en esclaves les colonies espagnoles, est en effet signé avec la Compagnie française de l'Asiento ou de Guinée, chargée de fournir des esclaves aux colonies espagnoles et cela suscite l'inquiétude des autres nations ${ }^{14}$. Au cours de la guerre, les Bourbons se résignent à abandonner les domaines européens de l'Espagne mais parviennent à sauvegarder les communications avec l'Amérique espagnole. De toute évidence, lorsque la longue guerre européenne, voire mondiale, met les Bourbons en difficulté, l'arrivée de l'argent américain est pour eux une véritable bouffée d'oxygène.

\section{UNE NÉGOCIATION AU QUOTIDIEN}

Néanmoins, le conflit, en se prolongeant, montre aussi, face aux tentatives françaises, l'indépendance et les résistances de l'administration espagnole qui, dans des négociations très âpres, maintient la singularité du système de la Carrera de Indias.

Dans quel contexte se font ces discussions? Un dialogue s'établit à distance entre les deux rois même si on peut supposer que des ministres ou des conseillers contribuent à élaborer les lettres des deux princes ${ }^{15}$. La princesse des Ursins conseille la jeune reine d'Espagne et son mari. Elle contribue à maintenir une relation étroite avec Versailles tout en informant la cour de France des réalités qui s'imposent à Philippe V. Les ambassadeurs de France participent au gouvernement espagnol mais les conflits éclatent aussi avec les ministres et les conseils espagnols ${ }^{16}$. Il faut attendre Amelot pour qu'une meilleure harmonie s'établisse ${ }^{17}$. La France a également sur place Daubenton, représentant de Pontchartrain et du département de la Marine, pour défendre les intérêts français: cet "agent général" a la charge de rassembler les informations transmises par les différents consuls français ${ }^{18}$. Le gouvernement français

13 Dahlgren, E., W.: Les relations commerciales et maritimes entre la France et les côtes de l'Océan Pacifique, I, Le commerce de la mer du Sud jusqu'à la paix d'Utrecht, Paris, Champion, 1909.

14 Villiers, P., JacQuin, P., RAGON, P.: Les Européens et la mer: de la découverte à la colonisation (14551860), Paris, Ellipses, 1997.

15 Bernardo Ares, J. M., Echeverría Pereda, E., Ortega Arjonilla, E.: De Madrid a Versailles. La correspondencia bilingüe entre el Rey Sol y Felipe V durante la guerra de sucesión, Barcelona, Planeta, 2011. Voir aussi Désos, C.: Les Français de Philippe V. Un modèle nouveau pour gouverner l'Espagne, 1700-1724, Strasbourg, Presses universitaires de Strasbourg, 2009.

16 BÉLY, L.: La présence et l'action des ambassadeurs de France dans le gouvernement de Philippe $V$ d'Espagne: conduite de la guerre et négociation de la paix, en Molinié, A., Merle, A.: L'Espagne et ses guerres, Paris, PUPS, 2004, pp. 183-201.

17 Hanotin, G.: “Au service de deux rois: l'ambassadeur Amelot de Gournay et l'union des couronnes", thèse soutenue à l'Université Paris-Sorbonne, 3 décembre 2011.

18 Cette fonction nouvelle fait l'objet d'une recherche doctorale de Sylvain Lloret 
doit néanmoins envoyer un spécialiste du grand commerce, Nicolas Mesnager, député du commerce de Rouen, pour discuter avec les autorités espagnoles. Mesnager ${ }^{19}$.

Lors d'une première négociation, de décembre 1704 à mai 1706, en Espagne, Mesnager obtient une nouvelle organisation du commerce: seuls les "étrangers alliés à l'Espagne" pourront envoyer des marchandises vers l'Amérique, ce qui doit favoriser les négociations en incitant les ennemis à faire la paix avec l'Espagne. Le contexte commercial semble favorable alors aux Français. Il faut bien constater un véritable démarrage économique des ports de l'Atlantique.

L'importance des Malouins, étudiés par André Lespagnol ${ }^{20}$, dans le commerce de la mer du Sud est également évidente: ils font une véritable "percée" dans l'empire espagnol mais choisissent essentiellement l'interlope, qui leur permet de contourner le monopole de Cadix. La question que pose André Lespagnol est de savoir s'ils veulent participer au commerce en Amérique ou bien partager le trésor américain. Les négociants malouins découvrent que le commerce direct dans la mer du sud (le Pacifique sud) est techniquement possible et financièrement bon. La démonstration étant faite en 1703, les négociants autour de Danycan se lancent dans cette aventure sans tenir compte des risques qu'elle fait courir aux relations franco-espagnoles. Le contrôleur général des finances de Louis XIV donne un soutien net et déterminé en 1705, même s'il dissimule ce négoce derrière des passeports accordés pour faire des découvertes. Le feu vert est donné à tous les interlopes, donc à une présence française dans les ports américains. Pire, à partir de 1712, des bateaux malouins passent à Cadix pour y compléter leur cargaison.

En arrivant d'Europe ou en quittant le Pérou, les marins français choisissent l'escale de Concepcion qui devient "la base logistique principale des interlopes français dans la mer du Sud" (André Lespagnol). Ils trouvent, près de la ville, à Talcahuano, un mouillage sûr, bien abrité des vents. Ils peuvent même y construire des bâtiments pour faire du commerce le long des côtes. Ce sont des installations semi-permanentes, avec des dortoirs, où les matelots peuvent retrouver la santé, et avec des entrepôts. En raison de séjours de plus en plus longs des bateaux et d'un phénomène de désertion, on aboutit à une forme de sédentarisation d'une population française. Un commissaire note en 1714 qu'à Concepcion, les officiers ont bâti des maisons comme s'ils devaient y passer toute leur vie ${ }^{21}$.

Ces Français se comportent comme "en pays de conquête", souligne le même observateur, qui déplore les débordements de la jeunesse. Des frictions ou des querelles éclatent avec les Espagnols installés dans la région. Cette ruée vers l'argent a conduit ainsi au développement d'une colonie française avec également des marins en relâche et des déserteurs. Les bateaux trouvent sur place du bois, de l'eau et des vivres, fournis par un riche arrière-pays agricole. Les Français bénéficient de la protection

19 Mesnager est lui aussi "une figure hybride entre un bureaucrate efficace et un homme du négoce", comme le consul espagnol à Rouen, José Paulo, étudié par Pradells Nadal, J.: "Observaciones sobre el commercio del cónsul José Pauló", en LoBo, op. cit., (nota 7), pp.119-125.

20 Lespagnol, A.: Messieurs de Saint-Malo. Une élite négociante au temps de Louis XIV, Saint-Malo, L'Ancre de Marine, 1990.

21 Ibidem, p. 602. 
des autorités coloniales, les Uztariz. Concepcion constitue enfin un observatoire privilégié et facilite donc le commerce sur les côtes américaines.

Cette expérience française devient un modèle: un trafic illégal ou quasi-illégal, un établissement très localisé pour permettre la circulation des bateaux, rassembler de l'information et soutenir le commerce. Une telle présence semble une manière commode de respecter en gros l'organisation espagnole tout en trouvant de nouveaux marchés en Amérique. Les Hollandais et les Anglais, dans les négociations, cherchent ensuite à obtenir de semblables avantages.

L'Angleterre profite par ailleurs de la guerre de Succession d'Espagne pour imposer au Portugal les accords Methuen, prolongeant dans le domaine économique le revirement diplomatique du Portugal qui entre dans la coalition contre les Bourbons ${ }^{22}$. Et Mesnager suppose que les puissances maritimes ont obtenu aussi des assurances du compétiteur de Philippe V, l'archiduc Charles ${ }^{23}$, leur promettant des avantages dans le commerce hispano-américain en cas de victoire.

\section{LA RECHERCHE D'INFORMATION ÉCONOMIQUE}

Le manuscrit de Jean de Monségur, publié par Jean-Paul Duviols, "Nouveaux Mémoires touchant le Mexique ou la Nouvelle-Espagne", révèle que le gouvernement français, surtout le secrétaire d'État de la Marine, Jérôme de Pontchatrain, cherche à glaner des informations exactes sur les marchés américains. Le voyageur apparaît bien comme un "soldat-diplomate-espion", selon la formule de Jean-Paul Duviols.

L'informateur s'intéresse au pays et à ses habitants, avant d'aborder les questions économiques. Il souligne que le Mexique connaît un mélange des populations de toutes origines: "Ces divers mélanges de toutes ces nations les unes avec les autres, composent tant de sortes de races qu'on en compte plus de quinze..." Cette vision raciale de la société débouche sur un jugement moral, mais aussi esthétique: "On peut dire qu'à la réserve des races des Espagnols de l'Europe, celle des créoles et des métis, toutes les autres sont vilaines et dégoûtantes et même affreuses à nos yeux" 24 . Monségur note que les enfants ont des nourrices choisies parmi les "négresses", les mulâtresses, quelquefois les métisses et les Indiennes: “...ainsi ce n'est pas une merveille de les voir toujours grossiers, ignorants et remplis des mauvaises inclinations qu'ils ont sucés avec le lait et la fréquentation d'une aussi pernicieuse race"25.

Monségur expose une maxime que l'Europe reconnaît volontiers: "Le commerce des Indes occidentales est le plus riche et le plus noble qui se fasse dans tout le monde. C'est par lui qu'il se fait la plus grande consommation de toutes les denrées

22 Cardoso, J. L., Cluny, I., Dores Costa, F. y otros: O Tratado de Methuen (1703): diplomacia, guerra, política e economia, Lisboa, Livros Horizonte, 2003.

23 Sur l'Archiduc, comme le désignaient les partisans de Philippe V, ou le roi Charles III, voir LEón SANZ, V.: Entre Austrias y Borbones: el archiduque Carlos y la monarquía de España, Madrid, Sigilo, 1993; LEóN SAnZ, V.: Carlos VI: el emperador que no pudo ser rey de España, Madrid, Aguilar, 2003.

24 Duviols, J. P. (ed.): Mémoires du Mexique. Le manuscrit de Jean de Monségur, 1707-1709, Paris, Chandeigne, 2002, p. 77.

25 Ibidem, p. 80. 
et de toutes les marchandises que fournissent l'Europe et une partie de l'Asie ${ }^{26}$." Il en veut pour preuve l'or, l'argent, les perles, les émeraudes, la cochenille et l'indigo qui sont passés en Europe. Ce commerce est le "plus grand de tous ceux qui se font sur la terre" et "le principe de l'action et du mouvement de tous les autres". Il en tire des conclusions: "Cela supposé, il est facile de voir qu'il n'y a aucun peuple, aucune puissance, ni aucun État dans le monde qui n'ait intérêt d'avoir part au riche commerce, et que les nations qui trouveront les moyens d'introduire en Amérique les marchandises et les denrées qui y trouvent plus de valeur, de débit et de consommation, seront celles qui profiteront de ses trésors et qui auront la meilleure part de ses richesses" 27 . Il constate alors: "L'Espagne et la France sont aujourd'hui en état de faire ce commerce le plus avantageusement qu'aucune autre puissance de la terre..." Pour cela, il faut que le roi d'Espagne "qui est le maître souverain de l'Amérique" y fasse régner plus d'ordre et de justice et qu'il permette à un plus grand nombre de ses sujets de s'y établir, en particulier pour travailler dans les mines. Monségur propose une véritable politique au chapitre XXXVII: “Que l'Espagne alliée de la France et unie avec elle peut elle seule fournir les Indes occidentales de toutes les marchandises qui s'y consomment et faire seule tout le commerce de l'Amérique". L'union des deux couronnes, que permet l'avènement de Philippe $V$, déboucherait donc sur une métamorphose du commerce colonial.

Le chapitre XLII décrit en détail le commerce de la Nouvelle-Espagne. Il montre comment des navires français, de Saint-Malo, de la Martinique et d'autres parties de la France se sont introduits "à la file avant et après la flotte de 1706". Ils profitent de ce que le Mexique manque de beaucoup de choses à ce moment-là. Le voyageur constate que le monopole se trouve aussi contourné par des trafics faits par des Espagnols de port en port et il mentionne La Havane, Maracaïbo, Caracas et Cumaná. Il signale même l'introduction de marchandises d'Angleterre et de Hollande. Après la flotte de 1706, arrive le galion à Acapulco: toutes les marchandises se vendent bien. Le Français décrit le système qui se met en place. Des bateaux français arrivent, surtout de Saint-Malo et de la Martinique, richement chargés. Ils ne peuvent décharger s'ils ne laissent pas leurs marchandises à des prix bas aux officiers royaux qui leur interdisent de vendre librement leur cargaison à des marchands. Il mentionne même un cas où les officiers royaux, ayant appris un marché avec un particulier, se saisissent des marchandises "et de l'argent jusqu'au coffre du capitaine qui fut mis en pièces" 28 . Telle est la leçon de cette présence des Français et de leur volonté de contourner les règles coloniales de l'Amérique: “...ces officiers ne voulurent plus accorder des permissions de décharger, qu'à condition de leur vendre à eux-mêmes, à des bas prix qu'ils fixaient à leur fantaisie, lesquels ils augmentaient aux particuliers à qui ils vendaient au moins $30 \%$ de bénéfices et souvent jusqu'à $40 \%$ et $50 \%$, de manière que ces entrées n'ont été proprement que pour les vice-rois et pour les ministres, ses émissaires, sans que les particuliers aient pu jouir des occasions d'acheter des mains des Français" 29 .

26 Ibidem, p. 97.

27 Ibidem, pp. 97-98.

28 Ibidem, p. 257.

29 Ibidem, p. 258. 
Monségur accumule les données les plus précises et des évaluations numériques pour cerner le commerce possible en Amérique. Par exemple, il donne la liste des principales marchandises nécessaires au Mexique: “...le fer, l'acier, la cire blanche, les fruits, les soieries, les dorures, les dentelles, les fils, les étoffes et ouvrages de laine, les épiceries, les papiers, les cartes, les chapeaux, la mercerie et les drogues pour la médecine" ${ }^{30}$.

\section{LES DISCUSSIONS COMMERCIALES AVEC LES HOLLANDAIS}

Accablée par les défaites, la diplomatie française choisit bientôt de placer les négociations avec ses ennemis sur ce même plan commercial. D'abord avec les Hollandais qui restent intransigeants, puis avec les Anglais qui, eux, sautent sur l'occasion.

Ces négociations nécessitent le recours à des spécialistes. Mesnager est chargé de mettre en ordre et en forme les propositions sur des sujets que les diplomates d'ordinaire maîtrisent mal. Il mène la négociation de décembre 1707 à mars 1708 en Hollande et il y est question de l'Amérique. Dans son esprit, il convient de dissiper “tous les soupçons" qui ont été répandus: le roi de France et le roi d'Espagne auraient voulu s'emparer absolument du commerce des Indes "pour en partager entre eux les dépouilles". Des concessions dans ce commerce, où toutes les nations étrangères seraient à égalité pour entasser des marchandises sur les bateaux espagnols, doivent au contraire conduire à la paix. Plus tard, le négociateur propose même de faire de Cadix une ville-entrepôt, "ville neutre et asile de toutes les nations de l'Europe".

A propos des Malouins, Mesnager affirme aux Hollandais, avec une parfaite mauvaise foi, que le roi n'a pas donné de congé pour cette sorte de commerce vers le Pérou: il s'agit simplement pour eux de s'enrichir, comme les Hollandais et les Anglais qui font du commerce vers la Nouvelle-Espagne et la Terre ferme à partir de Curaçao et de la Jamaïque. Bref, un partage de la contrebande. Il rappelle aux Hollandais qu'il n'est pas possible pour les rois de s'emparer des richesses des Indes occidentales, que les Américains ont un rôle à tenir dans le trafic "comme les autres peuples peuvent faire des fruits que la terre leur donne" et qu'ils échangent l'argent avec ceux qui leur apportent les "nécessités de la vie". Et ce commerce ne convient pas aux "têtes couronnées". "Que les fonds immenses qu'il y faudrait employer les mettraient hors d'état de le faire quand même ils voudraient l'entreprendre, que tout le fruit qu'ils en pourraient tirer ne serait qu'un profit de négociant qui serait toujours médiocre par rapport au capital et aux frais de régie, principalement pour des monarques qui voudraient le faire servir aux frais d'une guerre..." ${ }^{31} \mathrm{C}$ 'est insister sur le rôle de l'initiative privée et l'inefficacité des constructions étatiques, sur la place de la liberté et les méfaits des organisations royales. Cette vision libérale est une leçon de l'histoire après les espoirs placés dans les grands projets gouvernementaux.

\footnotetext{
30 Ibidem, p. 196.

31 Archives du Ministère des Affaires étrangères, Correspondance politique Hollande 214, fol. 52-54, Mesnager à Torcy, de La Haye, 24 janvier 1708.
} 
Lorsqu'il réfléchit à ce commerce en songeant aux intérêts français, Mesnager envisage des formes plus subtiles d'influence. Il insiste sur le rôle des modes vers l'Amérique: "Le principal moyen d'y trouver de l'avantage est qu'il plaise au roi engager le Roi Catholique à continuer ses sujets dans l'usage de nos habillements, et faire en sorte qu'il s'étende en Espagne de plus en plus: cet usage passera jusque dans les Indes, avec les officiers qui y retournent après avoir sollicité leurs emplois à Madrid. Cela produira infailliblement une consommation très considérable de toutes nos manufactures et de nos ouvrages en Espagne et dans l'Amérique, et la France en retirera une plus grande portion de ses richesses qu'elle n'a fait jusqu'à présent" ${ }^{32}$. La conquête des marchés se ferait ainsi, non par le biais du pouvoir politique et de la coercition, mais par l'inventivité manufacturière, le jeu de la mode et la séduction exercée par les produits. Le négociateur français propose donc de modeler un consommateur américain sur un modèle, inspiré aux Espagnols par le roi d'Espagne, lui-même prince d'origine française. Le souverain espagnol, comme son grand-père, devient le premier inspirateur des modes et, à travers sa personne, le soutien des manufacturiers et des marchands français.

La négociation française avec les alliés échoue en 1709 et 1710 lorsqu'elle est menée par le Grand Pensionnaire de Hollande. Les relations entre Louis XIV et Philippe $\mathrm{V}$ deviennent néanmoins difficiles car le premier se résigne à abandonner son petitfils, même s'il n'accepte pas de lui faire la guerre, comme le réclament les alliés. En 1709, des rumeurs circulent à propos des conférences de La Haye et persuadent Philippe V que le roi de France a l'intention de rappeler ses troupes. Il veut donc mettre des garnisons espagnoles dans les places ainsi abandonnées. L'ambassadeur Amelot se précipite au palais “...j'ai assuré ce Prince sur ma tête qu'il ne devait point craindre que le roi son grand-père retirât ses troupes sans l'en avertir auparavant et lui donner le temps de prendre ses mesures." Amelot ajoute: "Le roi d'Espagne, Monsieur, est dans une situation si cruelle qu'il ne faut pas s'étonner de ses inquiétudes [...] Nous nous trouvons dans des conjonctions bien épineuses dont il faut que nous tâchions de sortir avec honneur s'il est possible, et sans commettre le service et la réputation du roi notre maître" 33 . Le 13 juin, Amelot apprend que les conférences ont échoué, que les soldats français néanmoins quittent la péninsule et que lui-même doit partir: “...il a paru à S.M. que le roi d'Espagne, ayant dorénavant à se défendre par ses seules forces, serait obligé de mener le ministère sur un autre pied qu'il n'a été dans ces derniers temps et que par conséquent mon séjour en ce pays-ci ne saurait plus être utile au roi son petit-fils" ${ }^{34}$.

En 1711, la négociation aboutit à des préliminaires lorsque le gouvernement anglais s'en charge dans le plus grand secret. L'Angleterre ne demande pas de territoire pour elle-même dans l'immense empire espagnol, mais elle obtient l'asiento des esclaves et un vaisseau de permission. Elle souhaite aussi avoir des places en Amérique pour faciliter ce trafic et l'entrée de marchandises qu'il suppose. Le congrès d'Utrecht se

\footnotetext{
32 Archives du Ministère des Affaires étrangères, Correspondance politique Hollande 214, fol. 108-109, "Vues secrètes...".

33 Archives du Ministère des Affaires étrangères, Paris-La Courneuve, Acquisitions extraordinaires volume 9-24, Lettre d'Amelot du 10 juin 1709.

34 Ibidem, lettre du 3 juin 1709.
} 
réunit au début de 1712, mais les discussions piétinent, tant qu'une solution n'est pas trouvée en matière dynastique.

Après les victoires franco-espagnoles dans la péninsule Ibérique, Philippe V est plus solidement installé sur son trône. Comme il semble impossible que le nouvel empereur Charles VI soit aussi roi d'Espagne, comme les relations entre Louis XIV et son petit-fils se distendent, l'Europe, après dix ans de combats, accepte l'idée de voir Philippe V demeurer à Madrid.

Le roi de France négocie pour la maison de Bourbon avec ses ennemis, comme si ceux-ci considéraient évidente l'obéissance de Philippe V à l'égard de Louis XIV. Or, une certaine méfiance a grandi entre le grand-père et le petit-fils. Comme Louis XIV demande un plein pouvoir pour négocier au nom de son petit-fils et à sa place, Philippe V lui répond le 14 février 1712: "Si je diffère à vous envoyer le nouveau plein pouvoir que vous m'avez demandé, ce n'est pas que je ne croie que vous n'en usassiez comme le meilleur grand-père du monde qui ne veut que mon bien, mais je suis bien aise, avant de me déterminer à vous l'envoyer de voir les demandes préliminaires que les Hollandais auront faites..." ${ }^{35}$ Les liens familiaux -"le meilleur grand père du monde qui ne veut que mon bien"- servent de protestation formelle d'amour. En réalité, la mise en garde politique est claire: le plein pouvoir ne touche ni l'Espagne ni les Indes; il ne doit être montré qu' à la dernière extrémité; toute cession ne doit être faite que si on y était "forcé".

\section{LES NÉGOCIATIONS ENTRE LE GRAND-PÈRE ET LE PETIT FILS}

En France, la situation dans la famille royale est transformée par la mort du dauphin en 1711, du duc de Bourgogne et de son fils aîné en 1712. Philippe V se rapproche du trône de France. La question dynastique se pose donc de façon aiguë. Dans l'ordre de succession, seul un enfant né en 1710, le duc d'Anjou, le futur Louis XV, dont la santé semble précaire, précède Philippe V. La diplomatie anglaise se préoccupe de trouver un moyen d'empêcher la réunion des deux couronnes.

Louis XIV confie à Philippe V le 11 mars 1712: "Nous ignorons les secrets de la Providence, mais V.M. est présentement regardée de toute l'Europe comme prochain héritier de ma couronne. Je suis persuadé qu'au milieu de ces événements funestes, vous sentez plus de tendresse pour votre famille et, s'il est possible, que vous vous intéressez encore plus vivement au bien d'un royaume qui pourrait vous appartenir un jour." Ce discours ambigu rappelle à Philippe V qu'il est sur le point d'être l'héritier de la couronne de France et qu'il doit veiller aux intérêts de sa "famille" au nom de la tendresse qu'il a pour elle et pour son futur royaume.

La réponse vient d'Angleterre. Le gouvernement anglais imagine de régler la succession d'Espagne par le traité de paix. L'idée d'une renonciation de Philippe V à la couronne de France s'impose et il y renoncera pour lui-même et ses descendants. La succession de Louis XIV passera, à défaut du dauphin, au duc de Berry, ensuite au

35 Cité dans Bély, L.: La Société des princes, Paris, Fayard, 1999, pp. 337-341. Tous les textes qui suivent se retrouveront dans cet ouvrage Ils sont tirés de BAUDRILLART, op. cit., (nota 3), pp. 469-478. 
duc d'Orléans, puis aux autres princes du sang. Les Cortès d'Espagne donneront leur adhésion à cette solution. A l'extinction de la branche de Bourbon, elles choisiront une autre maison royale, sans doute la Savoie. L'Angleterre étant à la fois arbitre et médiatrice, la négociation se fait directement entre Londres, Versailles et Madrid.

Selon le ministre français Colbert de Torcy, il faut s'en tenir au testament de Charles II. Si Philippe V est appelé à la couronne de France, le duc de Berry le remplacera sur le trône d'Espagne; après lui, viendra le duc d'Orléans, puisque la renonciation d'Anne d'Autriche a été annulée en 1703. Louis XIV refuse d'envisager, au cas où Philippe V serait roi de France, d'attribuer l'Espagne à un de ses enfants, car les ennemis "objecteraient que la France et l'Espagne seraient toujours gouvernées par une même tête". C'était pourtant le plan proposé par la reine d'Espagne - Philippe V serait régent de France et roi d'Espagne, ou roi de France et régent d'Espagne.

La diplomatie anglaise refuse ces plans lointains et le secrétaire d'État anglais Saint-John veut une construction globale et rationnelle: "Nous voulons bien croire que vous êtes persuadés en France que Dieu seul peut abolir la loi sur laquelle le droit de votre succession est fondé, mais vous nous permettrez d'être persuadés, dans la Grande Bretagne, qu'un prince peut se départir de son droit par une cession volontaire et que celui en faveur de qui cette renonciation se fait, peut être justement soutenu dans ses prétentions par les puissances qui deviennent garantes du traité." Ainsi, la "cession volontaire", opposée au droit divin, fait intervenir une rationalité politique, garantie par les puissances européennes et les traités.

\section{LE CHOIX DE PHILIPPE V}

Louis XIV résume le choix simple qui est imposé à Philippe V, le 9 avril 1712: "Ou d'abandonner dès à présent l'Espagne et de venir auprès de moi pour jouir des droits qu'il n'aura peut-être jamais sur ma succession. Ou de renoncer à cette succession pour lui et ses descendants, et de conserver à ce prix l'Espagne et les Indes. Mon intention n'est pas de donner aucun conseil à ce sujet à mon petit-fils [...] je ne le presserai pas cependant pour le faire pencher à prendre un parti plutôt qu'un autre." Néanmoins, Louis XIV rappelle "ce qu'il se doit à soi-même, à ses enfants, à sa maison, à son royaume".

Un moment, on feint de croire que Philippe V pourra présenter le choix qu'il fera plus tard, au cas où le jeune Louis meurt avant son arrière-grand-père, ou si la succession de France vient à s'ouvrir et que Philippe y soit appelé: choisira-il alors l'Espagne ou la France? Ce choix virtuel permettait d'assurer l'avenir. Saint-John veut au contraire que le petit-fils de Louis XIV fasse son choix tout de suite et définitivement: “...qu'il vaut mieux et pour lui et pour nous que cette déclaration se fasse pendant le Congrès d'Utrecht que dans tout autre temps". Le gouvernement anglais cherche une solution évitant de nouvelles crises à l'avenir et rassemblant le plus grand nombre de garde-fous.

Face à l'insistance anglaise, il faut forcer la main de Philippe V. Le 18 avril 1712, Louis XIV a le sentiment que le roi d'Espagne restera fidèle à son nouveau pays: "J'ai peine à croire qu'un prince qui a régné pendant plus d'onze ans, qui aime ses sujets 
et qui a reçu tant de marques de leur fidélité, se résolve à les abandonner pour mener une vie privée dans l'attente incertaine d'une succession, la plus grande en vérité qui soit en Europe, mais dont l'espérance ne peut donner aucune autorité".

Devant les hésitations de son petit-fils, Louis XIV s'impatiente et utilise trois types d'arguments pour forcer Philippe V à prendre une décision immédiate. La renonciation à la couronne de France affermira Philippe V sur son trône, son autorité en deviendra plus absolue en Espagne et ses sujets lui seront plus fidèles. S'il refuse la renonciation, la guerre reprendra: le roi de France, considérant la paix comme absolument nécessaire, a donc l'intention de la faire le plus promptement qu'il lui sera possible. Le souverain français souligne enfin que Philippe V ne doit lui-même son trône qu'aux renonciations de son père et de son frère à leurs droits légitimes qu'ils tenaient de Marie-Thérèse d'Espagne.

En réalité, le roi d'Espagne a déjà pris sa décision, le 22 avril 1712: il est prêt renoncer à la succession de la couronne de France, s'il n'y a pas d'autre expédient et il laisse la diplomatie française choisir la procédure la mieux adaptée. Pour compenser ce sacrifice, Philippe V voudrait des concessions territoriales. Louis XIV en dit la vanité et essaie de faire oublier les menaces à peine voilées que ses lettres successives ont exprimées.

En mai 1712, une dernière proposition anglaise vient tout remettre en question. Au duc de Savoie, iraient l'Espagne et les Indes; à Philippe V, le Piémont, la Savoie, avec la Sicile, et ses droits sur la couronne de France seraient reconnus. S'il devenait roi, il ajouterait Savoie, Piémont, Montferrat, Nice, à la France, et la Sicile reviendrait à l'Autriche. Louis XIV se déclare favorable à cette solution inattendue: "Il [Philippe V] n'est point regardé comme étranger, il établit sa résidence presque sur la frontière de mon royaume, il est en état d'être promptement instruit de mes affaires, de venir me voir..." Il l'écrit aussitôt à Philippe V: "Jugez en effet du plaisir que je me ferais de pouvoir me reposer sur vous pour l'avenir, que si le dauphin vit, je laisserais en votre personne un régent accoutumé à commander..."

En même temps, il confie une lettre accablante à l'ambassadeur en Espagne, Bonnac, pour presser les événements: "Ne vous étonnez donc pas si vous apprenez que je fais la paix sans vous aux conditions que mes ennemis me proposent." Bonnac obtient une audience le 24 mai 1712 et les discussions ont lieu le 29 mai 1712. Bonnac affronte d'abord la princesse des Ursins (d'Aubigny, son confident, étant présent), puis Philippe V. Ce dernier a pris sa résolution, comme l'atteste sa lettre du même jour: “En m'en tenant à la résolution que j'ai déjà prise, je donne par là également la paix à la France et je lui assure pour alliée une monarchie qui, sans cela, pourrait un jour, jointe à ses ennemis, lui faire beaucoup de peine." Louis XIV accepte ce choix, par une lettre du 12 juin: "La résolution qu'il a prise est pour toujours, et quoi qu'il arrive, il doit compter que le traité de paix étant fait, il ne sera plus question de cet échange."

Le roi d'Espagne rend publique sa décision par une proclamation royale du 8 juillet 1712. Des rumeurs circulent immédiatement, selon lesquelles la renonciation serait conditionnelle et exclurait la branche d'Orléans, mais il semble bien difficile que Philippe $\mathrm{V}$ puisse, malgré ses rancunes, introduire de telles dispositions. Bonnac juge la renonciation future, dans une lettre à Torcy du 8 août: "Il se trouve ici des gens, 
comme en France, qui prétendent qu'elle sera nulle. Je ne suis point de ce sentiment et, si je crois que dans l'avenir elle peut être l'occasion de quelque guerre entre les deux royaumes, cela me parait fort éloigné et fort incertain. De plus, cette renonciation ou changement dans l'ordre du gouvernement n'est pas sans exemple."

Beaucoup plus tard, alors qu'il s'apprête à rompre ses engagements, lors d'une maladie du jeune Louis XV, Philippe V raconte, non sans angoisse, ce qu'ont été ses sentiments en 1712-1713:

Ces papiers regardent ma renonciation à la couronne de France. Je dois y ajouter que, doutant si la feue reine [sa première femme] et la princesse des Ursins préféreraient la France à l'Espagne et voudraient me conseiller de prendre ce parti, je promis et je crois même fis voeu de choisir l'Espagne, et je ne me souviens pas bien si même de ne jamais aller en France, et cela de mon propre choix, inclination et avec ferme intention de le faire, devant le Saint-Sacrement qui était exposé et en communiant, faisant même intention de communier pour promettre cela à Dieu; et communiant dans cette intention. Je demande si, après tout cela, je puis travailler à succéder à la couronne de France en cas que le dauphin vînt à mourir. J'ajoute encore que j'étais si porté à demeurer en Espagne, quand je fis la promesse ou le voeu, que je fus deux ou trois jours dans de grandes agitations et inquiétudes et même colère intérieure dans la crainte qu'on ne me conseillât le contraire ${ }^{36}$.

La confession de Philippe V montre que son choix se fonde sur un attachement profond pour son nouveau royaume: il a craint d'être forcé de l'abandonner.

\section{LES RENONCEMENTS DE PHILIPPE V}

Dans la négociation, Milan, la Sardaigne et Naples échappent à l'Espagne, les PaysBas aussi, promis également à l'empereur. Minorque offre une base à l'Angleterre en Méditerranée qui ne songe pas à rendre non plus Gibraltar. La redistribution des terres s'est faite sur les bases de la conquête. La paix vient donc en grande partie confirmer les résultats des opérations militaires. La France en revanche récupère Lille et les négociateurs ne semblent guère intégrer le sort des Catalans dans leurs savants calculs $^{37}$.

Des combinaisons subtiles permettent de récompenser des princes fidèles ou de leur faire oublier leurs malheurs en leur offrant des fragments de l'empire espagnol. Pour consoler Max Emmanuel de ses années d'exil, l'idée circule de lui donner la Sardaigne avec le titre de roi. Un des agents de l'Électeur méprise cet avantage. Torcy défend cette solution, dans une lettre à Bolingbroke, soulignant que, malgré la distance et le peu de liaison entre la Sardaigne et la Bavière il n'y a nulle incompatibilité que le même prince possède l'un et l'autre. Mais le ministre, en décembre

\footnotetext{
36 Baudrillart, A.: " Les prétentions de Philippe V à la Couronne de France", Revue des questions historiques, 41, (1887), pp. 96-149.

37 Albareda Salvadó, J.: El "cas dels Catalans". La conducta dels aliats arran de la guerra de successió (1705-1742), Barcelona, Pagès, 2005.
} 
1712, Torcy avoue à Polignac, l'un des plénipotentiaires à Utrecht que la Sardaigne seule n'est rien. Le territoire n'est ici qu'un simple nom, destiné à satisfaire, par une titulature, les ambitions dynastiques d'un prince: ni sa réalité géographique, ni ses caractères économiques et sociaux n'entrent en compte.

La Sicile est réservée par Londres à Victor-Amédée II de Savoie. Philippe V ne se résigne pas à abandonner toute présence espagnole en Italie; il veut pour cela retarder les concessions: “... ainsi il paraît qu'il convient au bien public de laisser à l'Espagne quelque portion de l'Italie du moins la Sicile pour qu'elle puisse protéger par là les Princes d'Italie contre le pouvoir exorbitant de la Maison d'Autriche. Les États qui m'y appartiennent sont assez beaux pour que vous puissiez être bien persuadé qu'il faut un bien aussi grand et aussi nécessaire que celui de la paix pour m'obliger à les sacrifier, et je n'ai été dans l'intention de le faire qu'à la dernière extrémité" 38 .

Grâce à l'engagement de Philippe V, la négociation avance et l'Angleterre abandonne la coalition ce qui facilite la victoire française à Denain en juillet. Un incident, à Utrecht, entre Mesnager et un diplomate hollandais, permet de geler toutes les négociations dans cette ville et de continuer les discussions bilatérales (août 1712).

Pour les accélérer, le secrétaire d'État Saint-John, devenu vicomte Bolingbroke, vient à Fontainebleau et discute avec le ministre français Torcy, surtout à propos de la Savoie - le duc deviendrait roi de Sicile - et de la Bavière - l'électeur deviendrait roi de Sardaigne. On veut aussi à Londres que la Sicile soit remise rapidement au nouveau roi. Il faut éviter qu'il ne cherche à l'échanger contre un territoire plus proche. La cession de la Sicile signifie un grand sacrifice de la part de l'Espagne car ce royaume n'a pas été conquis par les alliés et il a la réputation d'un territoire riche.

Un article secret de l'accord franco-anglais d'août 1712 accorde donc cette île à Victor-Amédée II et le ministre français craint la résistance espagnole: "M. le comte Bergheik, ministre de Philippe V, m'avait dit une heure auparavant qu'il avait ordre du Roi Catholique d'insister le plus longtemps qu'il lui serait possible sur la conservation de la Sicile [...] J'avertis M. de Bolingbroke qu'il devait s'attendre à quelques difficultés de la part des Ministres d'Espagne mais qu'elles ne seraient que pour la forme et qu'elles n'empêcheraient pas la conclusion" ${ }^{39}$. Les diplomates français considèrent donc que l'Espagne ne fera pas de résistance. Néanmoins, la monarchie hispanique accepte mal cette amputation volontaire au moment où tout l'empire espagnol se disloque ${ }^{40}$.

Mesnager laisse entendre que la Sicile permet aux Anglais de payer au souverain piémontais des subsides en retard. Rossi, un agent italien au service de Louis $\mathrm{XIV}$, met en garde le gouvernement français contre les dangers que peut faire courir en Sicile un changement de souveraineté ${ }^{41}$. Il connaît, dit-il, le pays, pour avoir été capitaine des gardes du cardinal del Giudice. Selon lui, celle-ci n'est attachée ni à

38 British Archives, State Papers 103/98, Papiers du maréchal d'Huxelles, fol. 274-5, copie d'une lettre de Philippe V à Louis XIV, 14 février 1712.

39 Mémorandum de Torcy, 19 août 1712, Archives du Ministère des Affaires étrangères (Paris), Correspondance politique, fol 193-207, ici fol. 203.

40 Álvarez-Ossorio, A., García García, B. J., León Sanz, V. (eds.): La pérdida de Europa. La Guerra de Sucesión por la Monarquía de España, Madrid, Fundación Carlos de Amberes, 2007.

41 Pour les références à ces textes, on se reportera à BÉLY, op. cit., (nota 11), pp. 529-530. 
Victor-Amédée II, ni à un autre souverain, mais elle tient à ses seuls intérêts. Rossi se demande si, profitant de l'échange, les Siciliens ne feraient pas appel aux Allemands. Torcy utilise l'argument dans son face-à-face avec Bolingbroke en août 1712: “...que les peuples de cette île naturellement portés aux révolutions auraient peine à demeurer tranquilles, lorsqu'ils se verraient abandonnés, et cédés par le roi d'Espagne à un nouveau maître..."

Le diplomate savoyard Del Borgo confie à Rossi que Victor-Amédée II compte se rendre en Sicile pour découvrir son nouveau royaume et pour se faire connaître de ses peuples. Torcy répète ses mises en garde: “...il s'expose à perdre son Royaume de Sicile; car il y a beaucoup d'agitation dans cette île et les habitants naturellement inquiets, disent assez hautement qu'il vaut mieux se choisir au maître et se donner à la Maison d'Autriche que de souffrir qu'on dispose d'eux comme d'un troupeau de moutons". Le ministre de Louis XIV définit paradoxalement un véritable "droit des peuples à disposer d'eux-mêmes".

La négociation anglo-espagnole peut continuer à Madrid sur les bases fixées à Londres. Le traité d'asiento ${ }^{42}$, fixant un minimum d'esclaves par an, pour trente ans, est signé le 26 mars 1713 à Madrid pour une compagnie anglaise, la South Sea Company. Les avantages commerciaux obtenus par la diplomatie anglaise ne concernent pas réellement des affaires d'État, mais des affaires privées.

Le renoncement de Philippe V à la Sicile et les renonciations du 5 novembre 1712 permettent au gouvernement anglais de convaincre des puissances moyennes de le suivre dans sa démarche de paix. L'Angleterre ne s'engage donc pas dans une paix séparée: elle entraîne avec elle la Savoie bien sûr, mais aussi le Portugal, la Prusse et les Provinces-Unies: les premiers traités sont signés le 11 avril 1713. L'empereur et la plupart des princes d'Empire restent en guerre et doivent mener une nouvelle campagne militaire contre la France.

Les plénipotentiaires espagnols ayant enfin obtenu l'autorisation de se rendre à Utrecht, ils peuvent signer à leur tour la paix: Philippe V cède Minorque et Gibraltar à l'Angleterre (13 juillet 1713) et, le même jour, la Sicile à Victor-Amédée II de Savoie. Ce dernier part pour Palerme où il se fait bientôt sacrer et couronner, mais il n'est reconnu ni par l'empereur, ni par le pape. La négociation est ralentie avec les Hollandais parce que Philippe V souhaite obtenir une principauté pour la princesse des Ursins qui serait ainsi devenue souveraine. Louis XIV doit menacer de ne pas aider Philippe $\mathrm{V}$ dans la reconquête de Barcelone pour qu'il signe à la fin avec les Provinces-Unies (26 juin 1714). Nous retrouvons une nouvelle négociation entre le roi de France et le roi d'Espagne à une étape cruciale ${ }^{43}$.

Les négociations entre les monarchies française et espagnole, entre Français et Espagnols, se situent à plusieurs niveaux et dans des espaces différents. Les rela-

42 On se reportera à l'ouvrage classique de Scelle, G.: La traite négrière aux Indes de Castille, contrats et traités d'assiento. Étude de droit public et d'histoire diplomatique puisée aux sources originales et accompagnée de plusieurs documents inédits, Paris, 1906. Voir aussi PaUl, H. J.: The South Sea Bubble. An Economic History of Its Origins and Consequences, London, Routledge, 2011.

43 Albareda Salvadó, J.: La Guerra de successió i l'onze de setembre, Barcelona, Empúries, 2000; Albareda Salvadó, J.: Felipe V y el triunfo del absolutismo. Cataluña en un conflicto europeo (1700-1714), Barcelona, Generalitat de Catalunya, 2002. 
tions entre les deux rois s'accompagnent d'échanges par des filières parallèles, les correspondances de la princesse des Ursins ou des ambassadeurs. À Madrid, les agents français doivent tenir compte des instances gouvernementales, le despacho et les conseils. Ils peuvent s'appuyer sur le réseau des consuls. À Versailles, les agents espagnols ont un interlocuteur privilégié, le secrétaire d'État des affaires étrangères, mais le département de la Marine s'intéresse aussi aux affaires commerciales. Dans les discussions internationales, la diplomatie française parle au nom des Bourbons, laissant vivre une construction impériale: le grand-père, chef de la maison, s'engage au nom de son petit-fils cadet.

Il faut aussi tenir compte de la société espagnole face aux drames qu'elle vit. Les Grands d'Espagne ont placé leur confiance dans la solution française. Certains sont vite déçus et se tournent vers Charles de Habsbourg. Beaucoup perdent les positions que l'immense empire espagnol en Europe pouvait leur assurer. Consuls et négociants français doivent aussi s'accommoder du système complexe de la Carrera de Indias. Ils cherchent des moyens de l'utiliser et de le contourner. Les marins et les marchands français s'engagent également en Amérique et ils doivent discuter là avec les autorités espagnoles qui peuvent leur permettre de faire des affaires ou les embarrasser.

Enfin, la paix d'Utrecht apparaît comme un aboutissement en mettant fin au conflit et en imposant une grande réorganisation géopolitique. C'est aussi une rupture. La plupart des pays européens sont mécontents des concessions qu'ils ont dû faire. La France ruinée cherche à se redresser et évite tout engagement dangereux en Europe. Louis XIV semble même se rapprocher de la cour de Vienne, tout en demeurant dans le sillage de l'Angleterre. En revanche, le roi d'Espagne n'a pas signé de traité avec l'empereur et il n'accepte pas les lourds sacrifices consentis en Italie. Le second mariage de Philippe V avec Elisabeth Farnèse ouvre de nouvelles perspectives. La disgrâce de la princesse des Ursins marque symboliquement un éloignement face à la tutelle politique française ${ }^{44}$. De plus la puissance espagnole trouve face à elle en Amérique des marchands anglais de plus en plus présents, ce qui ne peut que déboucher sur une tension permanente entre Madrid et Londres. Même avant la mort de Louis XIV, le lien entre Madrid et Versailles paraît désormais plus fragile.

\footnotetext{
44 Bély, L.: Élisabeth Farnèse et la princesse des Ursins: un coup de majesté?, en FraGNITO, G. (dir.): Elisabetta Farnese, principessa di Parma e regina di Spagna, Roma, Viella, 2009, pp. 71-89.
} 